\title{
SISTEMATIZACIÓN DE TRABAJOS DE GRADUACIÓN, UNA APROXIMACIÓN A LA GESTIÓN DEL CONOCIMIENTO
}

\author{
SYSTEMATIZING THESIS DEGREE, AN APPROACH \\ TO KNOWLEDGE MANAGEMENT
}

\author{
Sonia I. Mariño; simarinio@yahoo.com \\ Pedro L. Alfonzo; plalfonzo@hotmail.com \\ Maria V. Godoy; mvgodoy@exa.unne.edu.ar \\ Universidad Nacional del Nordeste, Argentina
}

\section{RESUMEN}

La Educación Superior proporciona diversos escenarios para implementar la Gestión del Conocimiento, siendo las TIC una herramienta clave. Se describe un sistema informático para administrar la información de trabajos de graduación, se detalla el modelo de proceso software y las funcionalidades asociadas a los distintos actores que interactúan. El conocimiento generado se transforma en valiosa información orientada a distintos actores del quehacer educativo, y se espera su proyección hacia otros ámbitos de la sociedad en la cual la Universidad tiene injerencia.

PALABRAS CLAVE: Gestión del conocimiento, educación superior, TIC, sistema informático, tesinas.

\begin{abstract}
Higher Education provides various scenarios for implementing Knowledge Management, being ICT a key tool. It is described a computer system to manage jobs graduation, software process model and the features associated with various actors interacting. The knowledge generated becomes valuable information aimed at various actors of educational work, and its projection is expected to other areas of society in which the University has interference.
\end{abstract}

KEYWORDS: Knowledge Management, higher education, ICT, computer system, thesis. 


\section{INTRODUCCIÓN}

El vertiginoso desarrollo de las tecnologías favoreció el paso de la sociedad industrial a la sociedad de la información y de ésta a la sociedad del conocimiento. Algunos autores diferencian entre sociedad de la información y sociedad del conocimiento.

La sociedad del conocimiento es un concepto amplio, referencia a la importancia de la ciencia y la tecnología en la generación y el desarrollo económico (Davenport y Prusak, 1998; Perez Lindo et al., 2005; Perez Lindo 2007; Perez Lindo 2007b). Se caracteriza por: el empleo intensivo de los sistemas de información y comunicaciones, el valor del conocimiento, la alfabetización en informática, el aprendizaje continuo y la organización electrónico-digital de la actividad humana (Perez Lindo et al., 2005; Carballo, 2006).

En la sociedad actual, el conocimiento es una variable determinante del desempeño de las organizaciones, su gestiòn es clave. Las instituciones de Educación Superior deben tratar con este paradigma y maximizar las potencialidades y las aplicaciones de las TIC en su desarrollo.

Cuando se aborda la tecnología desde la Gestión del Conocimiento (GC) es oportuno diferenciar entre: información y conocimiento. Mientras que la primera produce asociaciones necesarias para lograr la comprensión de los datos, el conocimiento facilita el fundamento para interpretar su comportamiento. Cuando la información es contextualizada y utilizada, es decir, cuando se presenta una relación de orden superior, se transforma en conocimiento (Passoni, 2005).

En Frost (2014) se exponen algunas claves que obstaculizan la GC. Entre las que se mencionan las tecnologías y como éstas se emplean. Siguiendo a Rodenes y Torralba (2006) el soporte informático hacia la GC debe incluir los siguientes objetivos básicos: aprovechar el conocimiento existente en la organización evitando redundancias, ayudar a crear nuevo conocimiento o innovar y aumentar continuamente la competencia y nivel de habilidad de las personas que trabajan en la organización.

Para implantar la GC en las organizaciones, es necesario crear, y mantener actualizadas las bases de información que representen el conocimiento existente de la información estructurada y no estructurada. Además, se requiere desarrollar y afianzar la infraestructura tecnológica necesaria para analizar inteligentemente los datos, compartir la información generada y re-crear, en forma cooperativa, el conocimiento.

Además, la infraestructura tecnológica corporizada en redes (Internet e Intranet), y necesaria para compatibilizar información interna y externa a la universidad y sistematizarla, debe favorecer flujos de información y comunicación de manera síncrona y asíncrona, entre los distintos claustros que componen la organización, ya sea en forma individual o grupal. Ésta debe facilitar los flujos de información y la comunicación interactiva entre las personas y grupos que integran la organización en 
todo momento. El problema consiste en saber cómo emplear las TIC para realizar una gestión eficaz del conocimiento.

Al igual que en otras organizaciones, las dedicadas a la Educación Superior deben maximizar las ventajas que brindan, las potencialidades y aplicaciones de las TICs. Los funcionarios y gestores deben contemplar el empleo en actividades de educación, investigación, extensión y administración; y además visualizar las alternativas de integración que otorga a su comunidad y ámbito de influencia a la Sociedad de la información y del Conocimiento.

Es así como la GC apunta a cubrir necesidades de la Educación Superior, siendo numerosas experiencias reportadas en este contexto (Passoni, 2005; García Peñalvo et al., 2010). Estas tratan a la gestión de la institución universitaria en sí, como en sus funciones de docencia, investigación y extensión (Perez Lindo et al., 2005, p. 264). En este trabajo se aborda la primera, dado que la implementación del sistema impactará en las funiones de la universidad.

Las carreras Licenciatura en Sistemas de Información, en sus respectivos planes de estudios -1999 y 2009-, describen un conjunto de conocimientos, habilidades y actitudes que definen el perfil de los graduados.

Trabajo Final de Aplicación (TFA) es la asignatura de la carrera Licenciatura en Sistemas de Información Plan 1999. La evolución de los conocimientos así como cuestiones curriculares nacionales se reflejaron en un nuevo plan de estudios identificado como Plan 2009 de la carrera Licenciatura en Sistemas de Información. Es así como surge Proyecto Final de Carrera (PFC), asignatura análoga a TFA del plan de estudios anterior.

Es una realidad la transición entre planes de estudios, motivo por el cual actualmente ambas asignaturas se encuentran vigentes siendo el plantel docente el mismo.

En las asignaturas TFA y PFC, el objetivo general es completar la formación académica y profesional de los alumnos, posibilitando la integración y utilización de los conocimientos adquiridos durante sus años de estudio para la resolución de problemas de índole profesional, académico y científico.

Por lo expuesto, estas asignaturas constituyen el espacio curricular, en el cual se generan los proyectos o tesinas. Una tesina o disertación de grado, siguiendo al Tesauro de la UNESCO consistiría en un diploma universitario de primer nivel.

En AUTOR1; AUTOR2 se detalló el contexto universitario que sustentó el diseño y el desarrollo del prototipo de sistema de información de los trabajos de graduación.

En este trabajo se describe el desarrollo de un prototipo funcional de un sistema de información orientado a la gestión de los datos derivados de los proyectos de tesinas elaborados en el contexto de las asignaturas TFA y PFC, ambas pertenecientes a los planes de estudios vigentes de la carrera Licenciatura en Sistemas de Información. 
Acordando con Muñoz y Mejia (2015, p.8) este sistema informático se constituye en una herramienta "para apoyar la automatización de la extracción de conocimiento tácito organizacional", transformándolo en conocimiento explícito que facilitará la construcción de indicadores.

\section{METODOLOGÍA}

La metodología aplicada se basó en las siguientes etapas:

Búsqueda y compilación de fuentes de datos previas conteniendo información generada en las asignaturas mencionadas.

* Revisión de un modelo de proceso incremental para el desarrollo del software.

* Elección de herramientas de desarrollo. En la construcción del prototipo para la gestión de conocimiento se seleccionó el lenguaje programación PHP y como gestor de base de datos MySQL. Cada uno de sus módulos funcionan de manera independiente o interdependiente. Esta característica otorga flexibilidad para la construcción de nuevas propuestas en función a la descripta en el trabajo.

* Adaptación del modelo de proceso para la gestión de datos generados en el contexto de las asignaturas mencionadas.

\section{MODELO DE PROCESO PARA LA GC DE TESINAS}

La razón de ser de las instituciones de Educación Superior tiene que ver con la GC desplegado en diversas situaciones. Polanyi (1996) identifica los siguientes conceptos vinculados a la GC:

* conocimiento tácito, es personal y de contexto específico, complejo de formalizar y comunicar; se adquiere en un contexto específico-práctico, surge de la experiencia y es subjetivo. En este sentido Muñoz y Mejía $(2015$, p.8) revisan los distintos tipos de conocimiento desde la mirada de referentes y consideran como "aquél conocimiento que no ha sido codificado y que presenta una mayor complejidad al transmitirse. En esta experiencia se corresponde con aquel proveniente de los alumnos que transitan la generación del proyecto de tesina, los docentes que los dirigen así como los docentes de las asignaturas que participan en la delimitación y definición de la idea final.

* conocimiento explícito, es un conocimiento codificado, puede transmitirse utilizando el lenguaje formal y sistemático, es también racionalizado y objetivo. El conocimiento tácito se formaliza en documentos requeridos por la asignatura, que luego se sistematizan en el sistema informático, es decir, éste último concentra y explicita el conocimiento obtenido de diversas fuentes.

Las tecnologías de la información dinamizan la transmisión de la información, sin embargo la gestión eficaz del conocimiento depende de la cognición humana y de la comunicación. Para lograrla, se necesita conocer y desarrollar una cultura de la 
información, así como ajustar los procesos de comunicación e información a las características específicas de las personas y grupos, denominado genéricamente como personalización de la información (Núñez Paula y Núñez Govín, 2005). En este sentido entre algunas acciones proyectadas sobre este particular se mencionan la divulgación del sistema informático en la comunidad educativa sobre la cual incidirá directamente: tanto en alumnos como en docentes y en equipos de I+D, dado que las producciones registradas podrán dar cuenta de los desarrollos en proceso o finalizados así como los actores o referentes de los mismos. Éstas acciones de difusión podrán exteriorizarse hacia otros destinatarios.

En el diseño y desarrollo tecnológico orientado a gestionar el conocimiento derivado de la administración de datos de los proyectos requeridos para la graduación, se aplicó como modelo de proceso el denominado evolutivo incremental (Pressmann, 2010). El despliegue de cada incremento, promueve evaluaciones que sustentan datos de realimentación. De este modo al realizar otro incremento, se avanza en la generación de la solución requerida.

A continuación se describen las etapas que componen cada incremento, del modelo de proceso software implementado (Fig. 1).

Etapa 1: Análisis del sistema de información para la gestión de tesinas de grado, integrando los conceptos previamente estudiados, seleccionados y adoptados para su utilización, con miras a su implantación.

* Análisis de factibilidad, de requerimientos funcionales y no funcionales del sistema, definición de limitaciones tecnológicas. Se definió el producto software y se determinó su factibilidad en el ciclo de vida desde la perspectiva de la relación costo-beneficio.

* Definición de seguridad en el acceso a la información. Los aspectos de seguridad en el acceso y manipulación de los datos, dieron lugar a establecer medidas para prevenir cualquier tipo de problemas tanto externos como internos.

* Especificación de requerimientos. Se detallaron las funciones solicitadas, se consideraron incrementos en porcentajes de la funcionalidad total del sistema.

* Selección de herramientas. Se optó por herramientas de libre distribución.

* Documentación Se elaboraron protocolos de soporte al análisis y diseño la solución propuesta.

Etapa 2: Diseño y desarrollo del sistema de información. Se realizaron las actividades concernientes a:

Diseño de las interfaces de comunicación con los usuarios.

* Construcción de las interfaces. Los diversos perfiles de usuarios a los cuales está dirigido.el productos de software determinaron su diseño.

* Construcción del prototipo. Consistió en elaborar el plan del prototipo, fijadas las restricciones con el usuario. Se refiere al análisis detallando cómo se trabaja, los módulos a implementar y las funciones a usar. 
Desarrollo. Se procedió a la codificación incremental y evolutiva de las versiones de los prototipos.

* Pruebas. Se procedió a la evaluación del prototipo con miras a realimentación. Asimismo, el despliegue de versiones, constituyó un medio de obtener datos para refinar el sistema, de este modo al final del proyecto el resultado cubrió los requerimientos.

* Documentación. Se elaboraron protocolos de soporte al desarrollo de la solución propuesta.

Etapa 3: Implementación y evaluación del sistema de información.

Migración de los datos. Se migraron los datos existentes desde antiguas fuentes de datos.

* Implementación. Como todo proceso de desarrollo de producto informático, se realizó la implementación.

* Evaluación del sistema. Se diseñaron y aplicaron instrumentos orientados a valorar el producto e implantaciones realizadas. Se construyeron aquellos más adecuados al tipo de información a reunir, en relación con los objetivos, recursos y población investigados.

* Fuente de información: Se dispuso de fuentes primarias de datos, consistiendo en archivos generados en las asignaturas.

* Resguardo de la información. Los datos almacenados y los códigos desarrollados se exportan regularmente con el propósito de asegurar la información institucional.

* Documentación. Se elaboraron protocolos de soporte a la implementación.

* Capacitación. Los integrantes de la asignatura son responsables de mantener actualizados los datos en el sistema desarrollado.

En la Fig. 2 se ilustra una composición de interfaces orientadas a sistematizar el registro y modificación de la información. Se dispone de un detalle de datos del alumno, el profesor orientador y del tribunal examinador. El seguimiento de los estudiantes permite identificar los distintos estados del proyecto, desde su inicio conteniendo el número de la resolución que lo avala hasta su defensa, concretándose así la graduación.

Las consultas disponibles ofrecen filtros para facilitar la búsqueda de la información de acuerdo a ciertos indicadores, entre los que se mencionan: proyectos aprobados en un periodo de tiempo, proyectos defendidos en un periodo de tiempo. Entre algunas consultas que apoyan el desempeño de los profesores se mencionan aquellas instancias en que actuaron como orientadores o participaron en tribunales examinadores, por ejemplo, información que aporta en la definicion de líneas de trabajos, informes particulares, entre otros. 


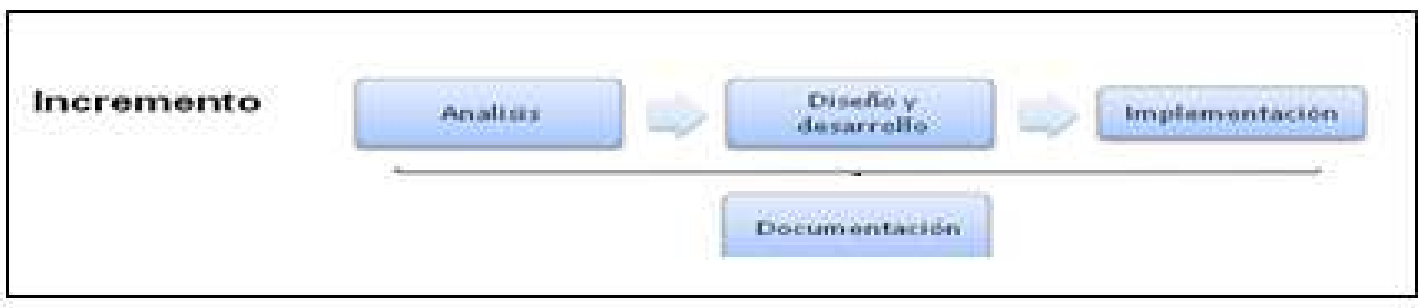

Fig. 1. Modelo de proceso.

El sistema permite la interacción con diferentes roles de usuarios con permisos según su perfil. Es así, como se controla el acceso y se gestionan las actividades según los roles asignados. Los usuarios deben autenticarse para acceder al sistema, excepto el usuario general, quienes visualizan unicamente datos públicos.

Los diferentes actores que interactúan con el sistema son:

* Administrador: usuario con privilegios de administración general de los datos, habilitado para definir otros niveles de acceso y usuarios. Accede al sistema con privilegios de configuración o modificación de parámetros. Es el responsable del mantenimiento del sitio. Otorga a los roles previstos sus respectivos permisos, es decir, qué operaciones puede realizar cada usuario, garantizando así la seguridad e integridad del mismo.

* Gestor de proyectos: usuario con permisos para crear, consultar y actualizar datos de los alumnos, relacionados con cualquier información relativa a los proyectos de trabajos finales. A modo de ejemplo se ilustra en la Fig. 2, las funcionalidades que permiten registrar los alumnos que regularizaron las asignaturas durante el cursado, como así también los que rindieron y aprobaron exámenes finales en condición de libres y los que solicitaron y fueron aceptados el cambio de tema del proyecto.

* Consultor de TFA/PFC: una vez iniciada la sesión especificando su nombre de usuario y contraseña, puede acceder a información derivada de los datos de los trabajos finales. A modo de ejemplo se menciona nómina de trabajos en los cuales participó como profesor orientador o como integrante del tribunal examinador, teniendo la opción de seleccionar un periodo de fecha determinado. Éste rol generalmente se asigna a los docentes de la facultad. Al elegir la opción "Actuó como orientador", se obtienen datos referentes al número de resolución del tema del proyecto, fecha de aprobación, tribunal examinador y el estado de la tesina (defendida o no), entre otros. Si el usuario optara por "Actuó como jurado", visualiza la fecha de resolución de la defensa y la fecha de defensa del proyecto; y los profesores coordinadores, entre otra información.

* Gestor de recursos: este perfil de usuarios dispone de permisos para publicar cualquier material útil para el taller de la asignatura, de manera de facilitar la disponibilidad a los alumnos que la adeudan. 
Alumno: se corresponde con un alumno de la asignatura. Es quien puede consultar datos de su proyecto y descargar materiales.

* Usuario General: es quien a través de la Web sin disponer de una cuenta en el sistema accede a consultas de datos públicos (Fig. 3). Como ejemplos se mencionan aquellas referentes a los listados de alumnos regulares y los graduados, y otra información vinculada a estas consultas.

Ruiz Tapia (2016, p. 29) mencionan cómo las TIC configuran nuevas relaciones comunicacionales en la sociedad, en las universidades y en los procesos de enseñanzaaprendizaje. La propuesta amplía ésta conceptualización dado el valor de las herramientas TIC en procesos de administración con miras a producir información valiosa.

Es así, como la sistematización de los datos de los proyectos de tesinas relevados son insumos en la construcción de indicadores para apoyar la toma de decisiones en contextos de Educación Superior.

Por ende, la implantación del sistema informático permitirá la construcción de información y de conocimiento. Para ejemplificar lo expuesto, se mencionan:

* las competencias de los sujetos que participan: los estudiantes que al realizar su trabajo de graduación se especializan en una temática disciplinar, así también los profesores orientadores y los evaluadores transmiten y explicitan determinados requerimientos que fortalecen la adquisición de conocimientos y competencias de los estudiantes. Generar información relacionada podría derivar en las principales competencias de equipos de I+D y de las organizaciones que propician la generación de éstas soluciones tecnológicas.

* la sistematización de los datos permitiría explicitar flujos de conocimientos internos y externos conformando o transformando redes de trabajo. Por ello, el capital de los sujetos involucrados en estos proyectos puede acrecentarse, siendo para los estudiantes alternativas de inserción laboral.

Por lo expuesto, en las interfaces se registran datos de los alumnos diferenciando si regularizan cursando o si rinden libres. Es menester ser alumno regular y contar con todas las asignaturas aprobadas para solicitar la evaluación y posterior defensa del trabajo integral de graduación.

Entre los principales campos que se registran y desde el cual se generan el seguimiento, reportes, estadísticas y se constituyen en insumo para la toma de decisiones se mencionan: datos personales del estudiante, plan de estudio al que pertenece (que vincula a la asignatura TFA o PFC), datos de contacto, datos de los profesores orientadores, tema propuesto, datos de los profesores que conformarán el tribunal examinador.

Esta información puede eliminarse o modificarse, dado que todo proyecto puede sufrir cambios, originados desde su desarrollo. 


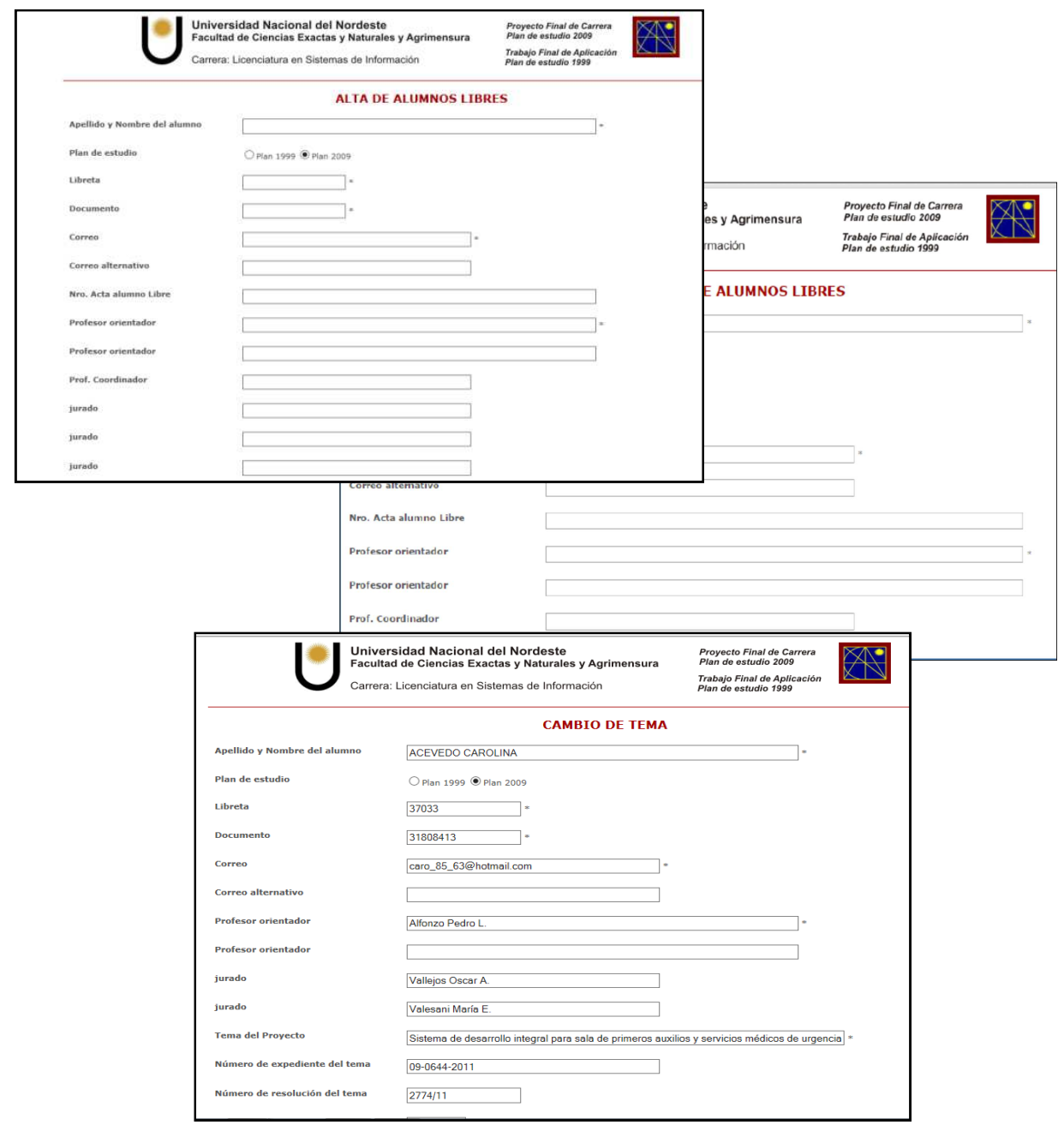

Fig. 2. Algunas funcionalidades disponibles para el Consultor de TFA/PFC. 


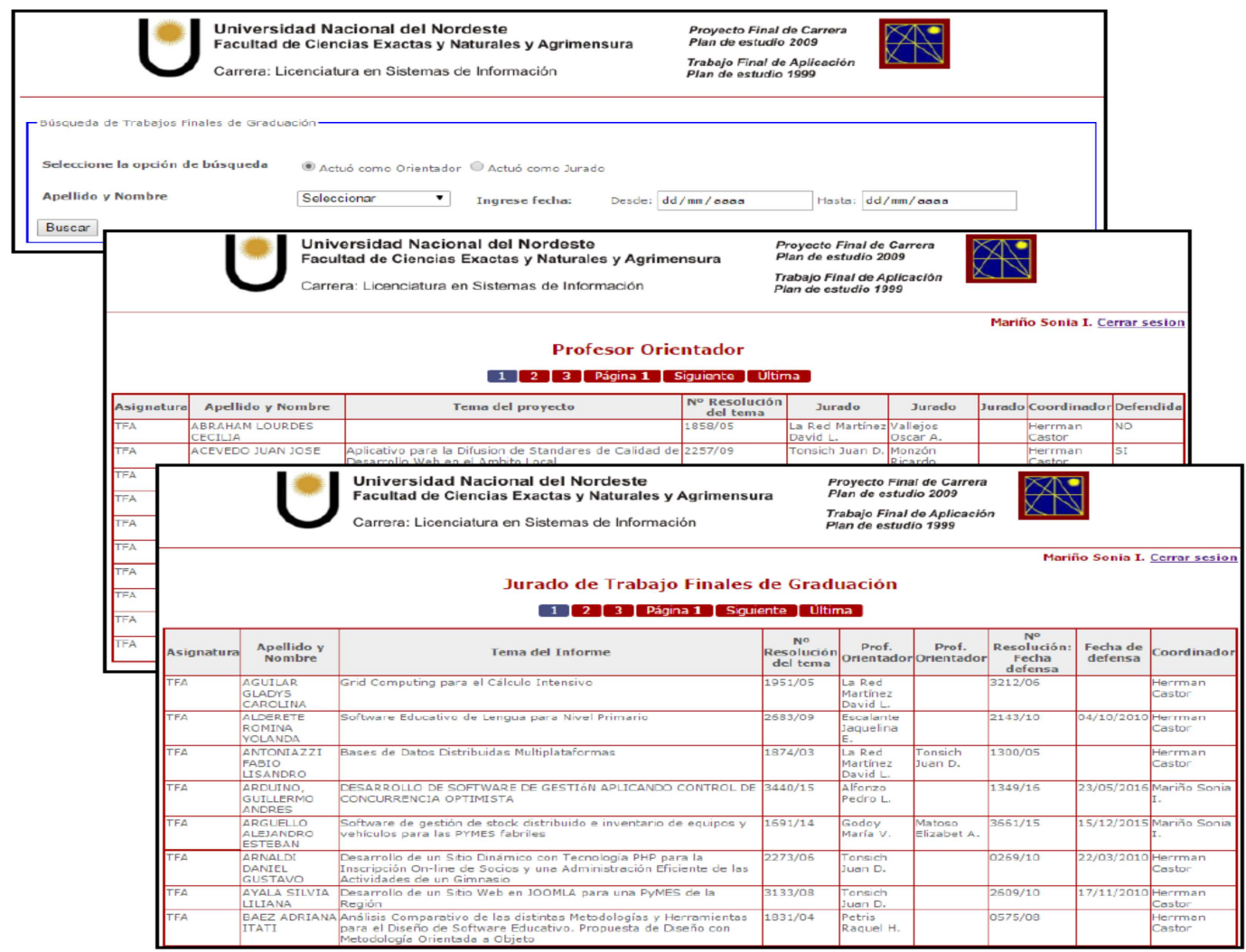

Fig. 3. Algunas funcionalidades disponibles para el Gestor de proyectos.

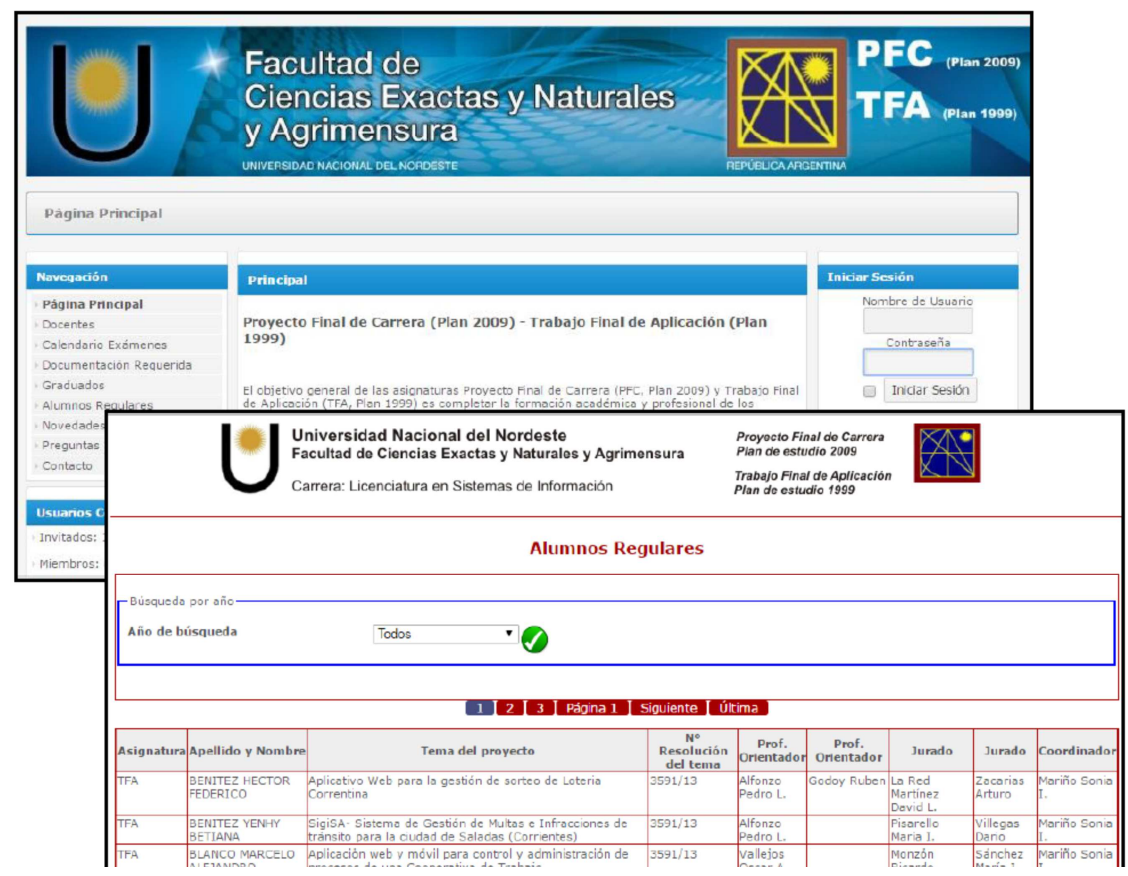

Fig. 4. Interfaz de consultas -perfil Usuario General- 


\section{CONSIDERACIONES FINALES}

La Educación Superior proporciona diversos escenarios para la implementación de la Gestión del Conocimiento. Las TIC y los sistemas de información impactan significativamente en las actividades humanas promoviendo el desarrollo de la sociedad del conocimiento. Las instituciones y organizaciones, principalmente las del ámbito educativo, deben adoptarlas con el propósito de avanzar y profundizar sus acciones de administración y difusión del conocimiento.

El artefacto descripto es una materialización software de la Gestión del Conocimiento para ámbitos educativos. En particular, éste sistema informático gestiona datos de las tesinas, es así como el conocimiento generado mediante su utilización se transforma en valiosa información orientada a distintos actores del quehacer educativo y de otros ámbitos de la sociedad en la cual la Universidad tiene injerencia.

Indudablemente su evolución será permanente, requiriéndose definir una "política del conocimiento" mediante un "plan de acción estratégico para el conocimiento" que contemple a la Institución y su relación de integración-interacción con la Universidad. Se retroalimentará incrementalmente mediante la incorporación de opciones definidas en función a los requerimientos de los potenciales usuarios.

Además, podrá adaptarse para su transferencia desde la Casa de Altos Estudios hacia otras unidades académicas, hacia organismos de gestión pública provincial con las adecuaciones que deberán contemplarse, acciones orientadas a aportar al desarrollo tecnológico de la región.

Como perspectivas futuras, se propone vincular el sistema con el sistema de alumnado de la Facultad, así como con la gestión de graduados, dado que al defender la tesina, se modifica el estado del alumno a graduado. Cabe aclarar que los datos registrados en esta soluciòn informática son complementarios a los dos sistemas previamente mencionados.

Además, se determinará el impacto de la propuesta entre los actores que utilizan el sistema informático en procesos de toma de decisiones: gestores universitarios, docentes, alumnos y otros sujetos que podrán interactuar con el mismo en procesos decisorios.

\section{REFERENCIAS}

CARBALLO, R. (2006). Innovación y gestión del conocimiento. Ed. Díaz de Santos.

DAVENPORT, T. y PRUSAK, L. (1998). Working Knowledge. Harvard Bussines School Press, Boston, Massachusett. 
FROST, A. (2014). A Synthesis of Knowledge Management Failure Factor. Recuperado el 22 de abril de 2016 de www.knowledge-management-tools.net

GARCía PeÑAlVo, F. J.; GARCíA DE FiguerolA, C., MERLO VEGA, J. A. (2010). Open Knowledge Management in Higher Education. 2010 [en línea] Disponible en: http://hdl.handle.net/10366/121874

MUÑOZ, M. y MEJIA, J. (2015). Tendencias en Tecnologías de Información y Comunicación, RISTI: Revista ibérica de Sistemas y Tecnologías de Información. No. 3.

NÚÑEZ PAULA I. A. y NÚÑEZ GOVÍN Y. (2005). Propuesta de clasificación de las herramientas - software para la gestión del conocimiento. Acimed 13(2). Recuperado el 22 de abril de 2016 de http://bvs.sld.cu/revistas/aci/vol13_2_05/aci03_05.htm.

PÉREZ LINDO A., RUIZ MORENO, L. VARELA, C., GROSSO, F., CAMÓS, C., TROTTINI, A, M, BURKE, M. L. y DARIN, S. (2005). Gestión del conocimiento. Un nuevo enfoque aplicable a las organizaciones y la universidad. Grupo Editorial Norma. Bs. As.

PÉREZ LINDO, A. (2007). De la revolución cognitiva a la gestión del conocimiento. Documento presentado en el curso de postgrado "Gestión del Conocimiento". Doctorado de Ciencias Cognitivas. Facultad de Humanidades. Universidad Nacional del Nordeste.

PÉREZ LINDO, A. (2007b). El Gobierno inteligente en la Republica Universitaria. De Platón a la Gestión del Conocimiento. Documento presentado en el curso de postgrado "Gestión del Conocimiento". Doctorado de Ciencias Cognitivas. Facultad de Humanidades. Universidad Nacional del Nordeste.

PASSONI, L. (2005) Gestión del conocimiento: una aplicación en departamentos académicos. Tesis, Recuperado el: 22-marzo-2016 de: http://www.accessmylibrary.com/coms2/summary_0286-31232685_ITM.

POLANYI, M. (1983) The Tacit Dimension. Gloucester, Massachussets. pp.1-25.

RODENES, A. y TORRALBA J. (2006), Sistema de ayuda a las decisiones en la gestión del conocimiento y las cooperativas. CIRIEC - Revista de Economía Pública, Social y Cooperativa. No. 49, pp. 55-75. España.

RUIZ TAPIA, J. A., MARTíNEZ, M., SÁNCHEZ, M. DE LA L. (2016). El impacto de las TICs en la calidad de la educación superior, Revista de Investigación en Ciencias Contables y Administrativas. Vol. 1(1), pp. 28-44. 


\section{Para citar este articulo:}

Mariño, S. I.; Alfonzo, P. L. \& Godoy, M. V. (2016). Sistematización de trabajos de graduación, una aproximación a la gestión del conocimiento. EDUTEC, Revista Electrónica de Tecnología Educativa, 57. Recuperado el dd/mm/aa de http://www.edutec.es/revista 